\title{
ASPECTS OF WEAK, $s$-CS AND ALMOST INJECTIVE RINGS
}

\author{
NASR A. ZEYADA AND AMR K. AMIN
}

\begin{abstract}
It is not known whether right $C F$-rings ( $F G F$-rings) are right artinian (quasi-Frobenius). This paper gives a positive answer of this question in the case of weak $C S(s-C S)$ and $G C 2$ rings. Also we get some new results on almost injective rings.
\end{abstract}

\section{INTRODUCTION}

A module $M$ is said to satisfy $C 1$-condition or called a $C S$-module if every submodule of $M$ is essential in a direct summand of $M$. Patrick F. Smith [20] introduced weak $C S$ modules. A right $R$-module $M$ is called weak $C S$ if every semisimple submodule of $M$ is essential in a summand of $M$. I. Amin, M. Yousif and N. Zeyada [1] introduced soc-injective and strongly soc-injective modules. Given two $R$-modules $M$ and $N, M$ is soc$N$-injective if any $R$-homomorphism $f: \operatorname{soc}(N) \longrightarrow M$ extends to $N . R$ is called right (self-) soc-injective, if the right $R$-module $R_{R}$ is soc-injective. $M$ is strongly soc-injective if $M$ is soc- $N$-injective for any module $N$. They proved that every strongly soc-injective module is weak $C S$.

N. Zeyada [24] introduced the notion of $s-C S$, for any right $R$-module $M, M$ is called $s-C S$ if every semisimple submodule of $M$ is essential in a summand of $M$.

A ring $R$ is called a right $C F$ ring if every cyclic right $R$-module can be embedded in a free module. A ring $R$ is called a right $F G F$ ring if every finitely generated right $R$-module can be embedded in a free right $R$-module. In section 2, we show that the right $C F$, weak $C S(s-C S)$ and $G C 2$ rings are artinian.

2010 Mathematics Subject Classification. 16D50.

Key words and phrases. $C S$, weak-CS, $S$-CS, rad-injective rings, almost injective rings, Kasch rings, Quasi-Frobenius rings.

Copyright (c) 2016 by ANUBIH. 
Zeyada, Hussein and Amin introduced the notions of almost and radinjectivity [23]. In the third section we make a correction to the result [23, Theorem 2.12] and we get a new result using these notions.

Throughout this paper $R$ is an associative ring with identity and all modules are unitary $R$-modules. For a right $R$-module $M$, we denote the socle of $M$ by $\operatorname{soc}(M) . S_{r}$ and $S_{l}$ are used to indicate the right socle and the left socle of $R$, respectively. For a submodule $N$ of $M$, the notations $N \subseteq^{e s s} M$ and $N \subseteq{ }^{\oplus} M$ mean that $N$ is essential and a direct summand, respectively. We refer to [2], [5], [7], [12] and [15] for all undefined notions in this paper.

\section{Generalizations of $C S$-modules and Rings}

Lemma 1. For a right $R$-module $M$, the following conditions are equivalent:

(1) $M$ is weak $C S$.

(2) $M=E \oplus T$ where $E$ is $C S$ with $\operatorname{soc}(M) \subseteq \subseteq^{\text {ess }} E$.

(3) For every semisimple submodule $A$ of $M$, there is a decomposition $M=M_{1} \oplus M_{2}$ such that $A \subseteq M_{1}$ and $M_{2}$ is a complement of $A$ in $M$.

Proof. (1) $\Longrightarrow(2)$. Let $M$ be a weak $C S$. Then $\operatorname{soc}(M)$ is essential in a summand, so $M=E \oplus T$ with $\operatorname{soc}(M) \subseteq{ }^{e s s} E$. Now if $K$ is a submodule of $E$, then $\operatorname{soc}(K) \subseteq \subseteq^{e s s} L$ where $L$ is a summand of $M$ and $L \subseteq \subseteq^{e s s}(K+L)$. But $L$ is closed, so $K \subseteq L$. Since $E \subseteq \subseteq^{e s s}(L+E)$ and $E$ is closed in $M$, so $L \subseteq E$ and $E$ is $C S$.

$(2) \Longrightarrow(1)$. If $E$ is $C S$ and a summand of $M$ with $\operatorname{soc}(M) \subseteq \subseteq^{e s s} E$, then every submodule of $\operatorname{soc}(M)$ is a summand of $E$ and a summand of $M$.

$(1 \Longrightarrow 3)$. Let $A$ be a submodule of $\operatorname{soc}(M)$. By (1), there exists $M_{1} \subseteq \oplus$ $M$ such that $A \subseteq \subseteq^{e s s} M_{1}$. Write $M=M_{1} \oplus M_{2}$ for some $M_{2} \subseteq M$. Since $M_{2}$ is a complement of $M_{1}$ in $M$ and $A$ is essential in $M_{1}$, then $M_{2}$ is the complement of $A$ in $M$.

$(3 \Longrightarrow 1)$. Let $A$ be a submodule of $\operatorname{soc}(M)$. By (2), there exists a decomposition $M=M_{1} \oplus M_{2}$ such that $A \subseteq M_{1}$ and $M_{2}$ is a complement of $A$ in $M$. Then $\left(A \oplus M_{2}\right) \subseteq{ }^{e s s} M=M_{1} \oplus M_{2}$ and $A \subseteq M_{1}$ then $A \subseteq{ }^{e s s} M_{1}$. Hence $M$ is weak $C S$ module.

Recall that, a right $R$-module $M$ is $s$-CS if every singular submodule of $M$ is essential in a summand [24].

Proposition 1. If $M$ is a right $R$-module, then the following conditions are equivalent:

(1) $M$ is $s$-CS.

(2) The second singular submodule $Z_{2}(M)$ is $C S$ and a summand of $M$.

(3) For every singular submodule $A$ of $M$, there is a decomposition $M=$ $M_{1} \oplus M_{2}$ such that $A \subseteq M_{1}$ and $M_{2}$ is a complement of $A$ in $M$. 
Proof. (1) $\Longleftrightarrow(2)$. [24, Proposition 14].

$(1) \Longleftrightarrow(3)$. A similar argument as in the proof of the above Lemma.

Given a right $R$-module $M$ we will denote by $\Omega(M)$ [respectively $C(M)$ ] a set of representatives of the isomorphism classes of the simple quotient modules (respectively simple submodules) of $M$. In particular, when $M=$ $R_{R}$, then $\Omega(R)$ is a set of representatives of the isomorphism classes of simple right $R$-modules.

Lemma 2. Let $R$ be a ring, and let $P_{R}$ be a finitely generated quasiprojective CS-module, such that $|\Omega(P)| \leq|C(P)|$. Then $|\Omega(P)|=|C(P)|$, and $P_{R}$ has finitely generated essential socle.

Proof. See [17, Lemma 7.28].

Proposition 2. Let $R$ be a ring. Then $R$ is a right PF-ring if and only if $R_{R}$ is a cogenerator and $R$ is weak $C S$.

Proof. Every right $P F$-ring is right self-injective and is a right cogenerator by [17, Theorem 1.56]. Conversely, if $R$ is weak $C S$ and $R$ is cogenerator then $R=E \oplus T$ where $E$ is $C S$ with $S_{r} \subseteq^{e s s} E$. By the above Lemma, $E$ has a finitely generated, essential right socle. Since $E$ is right finite dimensional and $R_{R}$ is a cogenerator, let $S_{r}=S_{1} \oplus S_{2} \oplus \ldots . \oplus S_{m}$ and $I_{i}=I\left(S_{i}\right)$ be the injective hull of $S_{i}$, then there exists an embedding $\sigma: I_{i} \longrightarrow R^{I}$ for some set $I$. Then $\pi \circ \sigma \neq 0$ for some projection $\pi: R^{I} \longrightarrow R$, so $(\pi \circ \sigma) \mid S_{i} \neq 0$ and hence is monic. Thus $\pi \circ \sigma: I_{i} \longrightarrow R$ is monic, and so $R=E_{1} \oplus \ldots \oplus E_{m} \oplus T$ where $\operatorname{soc}(T)=0$. So $R$ is a right $P F$-ring.

Proposition 3. [24, Proposition 16] Let $R$ be a ring. Then $R$ is a right $P F$-ring if and only if $R_{R}$ is a cogenerator and $\left(Z_{r}^{2}\right)_{R}$ is $C S$.

Proposition 4. The following conditions are equivalent:

(1) Every right $R$-module is weak $C S$.

(2) Every right $R$-module with essential socle is $C S$.

(3) For every right $R$-module $M, M=E \oplus K$ where $E$ is $C S$ with $\operatorname{soc}(M) \subseteq \subseteq^{e s s} E$.

Proposition 5. The following conditions are equivalent:

(1) Every right $R$-module is $s$-CS.

(2) Every Goldie torsion right $R$-module is $C S$.

(3) For every right $R$-module $M, M=Z_{2}(M) \oplus K$ where $Z_{2}(M)$ is $C S$.

Dinh Van Huynh, S. K. Jain and S. R. López-Permouth [11] proved that if $R$ is simple such that every cyclic singular right $R$-module is $C S$, then $R$ is right noetherian. 
Corollary 1. If $R$ is simple such that every cyclic right $R$-module is s-CS, then $R$ is right noetherian.

Proposition 6. If $R$ is a weak $C S$ and $G C 2$, and right Kasch, then $R$ is semiperfect.

Proof. Since $R$ is a weak $C S$, so $E$ is $C S$ by Lemma 1 and $R=E \oplus K$ for some right ideal $K$ of $R$ and so $E$ is a finitely generated projective module. By Lemma 2, $E$ has a finitely generated essential socle. Then, by hypothesis, there exist simple submodules $S_{1}, \cdots, S_{n}$ of $E$ such that $\left\{S_{1}, \cdots, S_{n}\right\}$ is a complete set of representatives of the isomorphism classes of simple right $R$-modules. Since $E$ is $C S$, there exist submodules $Q_{1}, \cdots, Q_{n}$ of $E$ such that $Q_{1}, \cdots Q_{n}$ is an direct summands of $E$ and $\left(S_{i}\right)_{R} \subseteq^{\text {ess }}\left(Q_{i}\right)_{R}$ for $i=1, \cdots, n$. Since $Q_{i}$ is an indecomposable projective and $G C 2 R$-module, it has a local endomorphism ring; and since $Q_{i}$ is projective, $J\left(Q_{i}\right)$ is maximal and small in $Q_{i}$. Then $Q_{i}$ is a projective cover of the simple module $Q_{i} / J\left(Q_{i}\right)$. Note that $Q_{i} \cong Q_{j}$ clearly implies $Q_{i} / J\left(Q_{i}\right) \cong Q_{j} / J\left(Q_{j}\right)$; and the converse also holds because every module has at most one projective cover up to isomorphism. It is clear that $Q_{i} \cong Q_{j}$ if and only if $S_{i} \cong S_{j}$ if and only if $i=j$. Thus, $\left\{Q_{1} / J\left(Q_{1}\right), \cdots, Q_{n} / J\left(Q_{n}\right)\right\}$ is a complete set of representatives of the isomorphism classes of simple right $R$-modules. Hence every simple right $R$-module has a projective cover. Therefore $R$ is semiperfect.

The following example shows that the proof given in [24, Proposition 13] is not true, since the endomorphism ring of an indecomposable projective module which is an essential extension of a simple module may be not a local ring. So we add an extra condition that $R$ is right $G C 2$ to prove the Proposition.

Example 1. Let $R$ be the ring of triangular matrices, $R=\left\{\left(\begin{array}{cc}a & 0 \\ b & c\end{array}\right): a\right.$ $\in Z, b, c \in Q\}$. Take $P_{1}=\left\{\left(\begin{array}{cc}a & 0 \\ b & 0\end{array}\right): a \in Z, b \in Q\right\}$ and $P_{2}=\left\{\left(\begin{array}{ll}0 & 0 \\ 0 & c\end{array}\right):\right.$ $c \in Q\}$, we see that $P_{1}$ is indecomposable projective module with simple essential socle and $P_{2}$ is a projective simple module. The socle of $P_{1}$ is isomorphic to $P_{2}$. Thus, the endomorphism ring of $P_{1}$ is isomorphic to $Z$ which is not local.

Proposition 7. If $R$ is right $s-C S$ and $G C 2$, and right Kasch, then $R$ is semiperfect.

Proof. Since $R$ is a weak $C S$, so $E$ is $C S$ by Lemma 1 and $R=E \oplus K$ for some right ideal $K$ of $R$ and so $E$ is a finitely generated projective module. By Lemma 2, $E$ has a finitely generated essential socle. Then, by hypothesis, 
there exist simple submodules $S_{1}, \ldots, S_{n}$ of $E$ such that $\left\{S_{1}, \ldots, S_{n}\right\}$ is a complete set of representatives of the isomorphism classes of simple right $R$ modules. Since $E$ is $C S$, there exist submodules $Q_{1}, \ldots, Q_{n}$ of $E$ such that $Q_{1}, \ldots Q_{n}$ is a direct summands of $E$ and $\left(S_{i}\right)_{R} \subseteq^{e s s}\left(Q_{i}\right)_{R}$ for $i=1, \ldots, n$. Since $Q_{i}$ is an indecomposable projective and $G C 2 R$-module, it has a local endomorphism ring; and since $Q_{i}$ is projective, $J\left(Q_{i}\right)$ is maximal and small in $Q_{i}$. Then $Q_{i}$ is a projective cover of the simple module $Q_{i} / J\left(Q_{i}\right)$. Note that $Q_{i} \cong Q_{j}$ clearly implies $Q_{i} / J\left(Q_{i}\right) \cong Q_{j} / J\left(Q_{j}\right)$; and the converse also holds because every module has at most one projective cover up to isomorphism. It is clear that $Q_{i} \cong Q_{j}$ if and only if $S_{i} \cong S_{j}$ if and only if $i=j$. Thus, $\left\{Q_{1} / J\left(Q_{1}\right), \ldots, Q_{n} / J\left(Q_{n}\right)\right\}$ is a complete set of representatives of the isomorphism classes of simple right $R$-modules. Hence every simple right $R$-module has a projective cover. Therefore $R$ is semiperfect.

Lemma 3. Let $R$ be a semiperfect, left Kasch and left min-CS ring. Then the following statements hold:

(1) $S_{l} \subseteq_{R}^{\text {ess }} R$ and $\operatorname{soc}(R e)$ is simple and essential in Re for all local idempotents $e \in R$.

(2) $R$ is right Kasch if and only if $S_{l} \subseteq S_{r}$.

(3) If $\left\{e_{1}, \ldots, e_{n}\right\}$ are basic local idempotents in $R$ then $\left\{\operatorname{soc}\left(R e_{1}\right), \ldots\right.$, $\left.\operatorname{soc}\left(R e_{n}\right)\right\}$ is a complete set of distinct representatives of the simple left $R$-modules.

Proof. See [17, Lemma 4.5].

Recall that a ring $R$ is right minfull if it is semiperfect, right mininjective, and $\operatorname{soc}(e R) \neq 0$ for each local idempotent $e \in R$.

Corollary 2. If $R$ is commutative $s-C S$ (weak $C S$ ) and Kasch, then $R$ is minfull.

Proof. Since every Kasch ring is $C 2$, so $R$ is semiperfect by Proposition 6 (Proposition 7). Thus using the above Lemma and [17, Proposition 4.3] $R$ is minfull.

Theorem 1. If $R$ is right weak $C S$ (s-CS), GC2 and every cyclic right $R$-module can be embedded in a free module (right $C F$ ring) then $R$ is right artinian

Proof. If $R$ is right weak $C S(s-C S)$ right $C F$, then by Lemma $1 R=E \oplus K$ where $E$ is $C S$ and $\operatorname{soc}(K)=0(Z(K)=0)$. Thus by Proposition 6 (Proposition 7), $R$ is semiperfect. The above Lemma gives $S_{r} \subseteq^{e s s} R_{R}$, so $K=0$. Hence $R$ is $C S$ and $R$ is right artinain by [9, Corollary 2.9].

Proposition 8. Let $R$ be a right FGF, right weak $C S$ (s-CS) and right $G C 2$ ring. Then $R$ is $Q F$. 
Proof. It is clear by Proposition 6 (Proposition 7), and [8, Theorem 3.7].

\section{Almost injective modules}

Definition 1. A right $R$-module $M$ is called almost injective, if $M=E \oplus K$ where $E$ is injective and $K$ has zero radical. A ring $R$ is called right almost injective, if $R_{R}$ is almost injective.

In [23], the statement of Theorem 2.12 is not true. The following Proposition is the true version of [23, Theorem 2.12]. Moreover, the rest of this section will be devoted to do the necessary changes of the related results in [23]. Also we get new results in view of the following Proposition.

Proposition 9. For a ring $R$ the following statements are true:

(1) $R$ is semisimple if and only if every almost-injective right $R$-module is injective.

(2) If $R$ is semilocal, then every rad-injective right $R$-module is injective.

Proof. (1). Assume that every almost-injective right $R$-module is injective, then every right $R$-module with zero radical is injective. Thus every semisimple right $R$-module is injective and $R$ is right $V$-ring. Hence, every right $R$-module has a zero radical. Therefore, every right $R$-module is injective and $R$ is semisimple. The converse is clear.

(2). Let $R$ be a semilocal ring and $M$ be a rad-injective right $R$-module. Consider a homomorphism $f: K \longrightarrow M$ where $K$ is a right ideal of $R$. Since $R$ is semilocal, there exists a right ideal $L$ of $R$ such that $K+L=R$ and $K \cap L \subseteq J$ [13]. Then there exists a $R$-homomorphism $g: R \longrightarrow M$ such that $g(x)=f(x)$ for every $x \in K \cap L$. Define $F: R \longrightarrow M$ by $F(x)=f(k)+g(l)$ for any $x=k+l$ where $k \in K$ and $l \in L$. It is clear that $F$ is a well-defined $R$-homomorphism such that $\left.F\right|_{K}=f$. i.e. $F$ extends $f$. Therefore $M$ is injective.

A ring $R$ is called quasi-Frobenius $(Q F)$ if $R$ is right (or left) artinian and right (or left) self-injective. Also, $R$ is $Q F$ if and only if every injective right $R$-module is projective.

Theorem 2. $R$ is a quasi-Frobenius ring if and only if every rad-injective right $R$-module is projective.

Proof. If $R$ is quasi-Frobenius, then $R$ is right artinian, and by Proposition 9 (2), every rad-injective right $R$-module is injective. Hence, every radinjective right $R$-module is projective. Conversely, if every rad-injective right $R$-module is projective, then every injective right $R$-module is projective. Thus, $R$ is quasi-Frobenius. 
Recall that a ring $R$ is called a right pseudo-Frobenius ring (right $P F$ ring) if the right $R$-module $R_{R}$ is an injective cogenerator.

Proposition 10. [17, Theorem 1.56] The following conditions are equivalent:

(1) $R$ is a right PF-ring.

(2) $R$ is a semiperfect right self-injective ring with essential right socle.

(3) $R$ is a right finitely cogenerated right self-injective ring.

(4) $R$ is a right Kasch right self-injective ring.

Theorem 3. If $R$ is right Kasch right almost-injective, then $R$ is semiperfect.

Proof. Let $R$ be right Kasch and $R_{R}=E \oplus T$, where $E$ is injective and $T$ has zero radical. If $J=0$, then every simple right ideal of $R$ is projective and $R$ is semiperfect (for $R$ is right Kasch). Now suppose that $J \neq 0$. Clearly, every simple singular right $R$-module embeds in $E$. In particular, every simple quotient of $E$ is isomorphic to a simple submodule of $E$, and so $E$ is a finitely generated injective and projective module containing a copy of every simple quotient of E. By [8, Lemma 18], $E$ has a finitely generated essential socle. Then by hypothesis, there exist simple submodules $S_{1}, \ldots, S_{n}$ of $E$ such that $\left\{S_{1}, \ldots, S_{n}\right\}$ is a complete set of representatives of the isomorphism classes of simple singular right $R$-modules. Since $E$ is injective, there exist submodules $Q_{1}, \ldots, Q_{n}$ of $E$ such that $Q_{1} \oplus \cdots \oplus Q_{n}$ is a direct summand of $E$ and $\left(S_{i}\right)_{R} \subseteq^{e s s}\left(Q_{i}\right)_{R}$ for $i=1,2, \ldots, n$. Since $Q_{i}$ is an indecomposable injective $R$-module, it has a local endomorphism ring. The projectivity of $Q_{i}$ implies that $J\left(Q_{i}\right)$ is maximal and small in $Q_{i}$. Then $Q_{i}$ is the projective cover of the simple module $Q_{i} / J\left(Q_{i}\right)$. Note that $Q_{i} \cong Q_{j}$ clearly implies $Q_{i} / J\left(Q_{i}\right) \cong Q_{j} / J\left(Q_{j}\right)$ and the converse also holds because every module has at most one projective cover up to isomorphism. But it is clear that $Q_{i} \cong Q_{j}$ if and only if $S_{i} \cong S_{j}$, if and only if $i=j$. Moreover, every $Q_{i} / J\left(Q_{i}\right)$ is singular. Thus, $\left\{Q_{1} / J\left(Q_{1}\right), \ldots, Q_{n} / J\left(Q_{n}\right)\right\}$ is a complete set of representatives of the isomorphism classes of the simple singular right $R$-modules. Hence, every simple singular right $R$-module has a projective cover. Since every non-singular simple right $R$-module is projective, we conclude that $R$ is semiperfect.

Proposition 11. The following conditions are equivalent:

(1) $R$ is a right PF-ring.

(2) $R$ is a semiperfect right rad-injective ring with $\operatorname{soc}(e R) \neq 0$ for each local idempotent e of $R$.

(3) $R$ is a right finitely cogenerated right rad-injective ring.

(4) $R$ is a right Kasch right rad-injective ring. 
(5) $R$ is a right rad-injective ring and the dual of every simple left $R$ module is simple.

Proof. (1) $\Leftrightarrow(2)$ By Proposition 9 (2).

(1) $\Rightarrow$ (3) Clear.

$(3) \Rightarrow(1)$ Since $R$ is a right rad-injective ring, it follows from [23, Proposition 2.5] that $R=E \oplus K$, where $E$ is injective and $K$ has zero radical. Since $R$ is a right finitely cogenerated ring, $K$ is a finitely cogenerated right $R$-module with zero radical. Hence, $K$ is semisimple. Therefore, by [22, Corollary 8], $R$ is a right $P F$-ring.

$(1) \Rightarrow(4)$ Clear.

(4) $\Rightarrow(1)$ If $R$ is right Kasch right rad-injective, then $R$ is right almostinjective ([23, Proposition 2.5]). Thus $R$ is semiperfect (3). Hence $R$ is injective by Proposition 9 (2). Therefore, $R$ is right $P F$.

$(1) \Rightarrow(5)$ Since every right $P F$-ring is left Kasch and left mininjective, the dual of every simple left $R$-module is simple by [16, Proposition 2.2].

$(5) \Rightarrow(1)$ By [23, Proposition 2.10], $R$ is a right min - CS ring (i.e. every minimal right ideal of $R$ is essential in a summand). Thus, by [10, Theorem 2.1], $R$ is semiperfect with essential right socle. Proposition $9(2)$ entails that $R$ is right self-injective, and hence right $P F$ by Proposition 10 .

$(1) \Longleftrightarrow(4)$ and $(1) \Longleftrightarrow(5)$ are direct consequences of [23, Proposition $2.5]$.

A result of Osofsky [18, Proposition 2.2] asserts that a ring $R$ is $Q F$ if and only if $R$ is a left perfect, left and right self-injective ring. This result remains true for rad-injective rings.

Proposition 12. The following conditions are equivalent:

(1) $R$ is a quasi-Frobenius ring.

(2) $R$ is a left perfect, left and right rad-injective ring.

Proof. (1) $\Rightarrow(2)$ It is well known.

$(2) \Rightarrow(1)$ By hypothesis, $R$ is a semiperfect right and left rad-injective ring. By Proposition 9 (2), $R$ is right and left injective, hence $R$ is quasiFrobenius.

Note that the ring of integers $Z$ is an example of a commutative noetherian almost-injective ring which is not quasi-Frobenius.

Definition 2. A ring $R$ is called right $C F$-ring ( $F G F$-ring) if every cyclic (finitely generated) right $R$-module embeds in a free module. It is not known whether right $C F$-rings ( $F G F$-rings) are right artinian (quasi-Frobenius rings). In the next result, a positive answer is given if we assume in addition that the ring $R$ is right rad-injective. 
Proposition 13. The following conditions are equivalent:

(1) $R$ is quasi-Frobenius.

(2) $R$ is right $C F$ and right rad-injective.

Proof. (1) $\Rightarrow$ (2) It is well known.

$(2) \Rightarrow(1)$ Since every simple right $R$-module embeds in $R, R$ is a right Kasch ring. By Proposition 11, $R$ is right self-injective with finitely generated essential right socle. Thus, every cyclic right $R$-module has a finitely generated essential socle, and by [21, Proposition 2.2], $R$ is right artinian, hence quasi-Frobenius.

Acknowledgement. The authors would like to thank the referee for valuable suggestions and comments.

\section{REFERENCES}

[1] I. Amin, M. Yousif and N. Zeyada, Soc-injective rings and modules, Comm. Algebra, 33 (2005), 4229-4250.

[2] F. W. Anderson and K. R. Fuller, Rings and Categories of Modules, Springer-Verlag, Berlin-New York, 1974

[3] J. E. Bjork, Rings satisfying certain chain conditions, J. Reine Angew. Math., 245 (1970), 63-73.

[4] V. Camillo, W. K. Nicholson and M. F. Yousif, Ikeda-Nakayama rings, J. Algebra, 226 (2000), 1001-1010.

[5] N. V. Dung, V. D. Huynh, P. F. Smith and R. Wisbauer, Extending Modules, Pitman Reasearch Notes in Math. Longman, 1994.

[6] C. Faith, Rings with ascending chain conditions on annihilators, Nagoya Math. J., 27 (1966), 179-191.

[7] C. Faith, Algebra II, Ring Theory, Springer-Verlag, Berlin-New York, 1976.

[8] J. L. G'omez Pardo and P. A. Guil Asensio, Essential embedding of cyclic modules in projectives, Trans. Amer. Math. Soc., 349 (1997), 4343-4353.

[9] J. L. Gómez Pardo and P. A. Guil Asensio, Rings with finite essential socle, Proc. Amer. Math. Soc., 125 (1997), 971-977.

[10] J. L. G'omez Pardo and M. F. Yousif, Semiperfect min-CS rings, Glasg. Math.J., 41 (1999), 231-238.

[11] Dinh Van Huynh, S. K. Jain and S. R. Lopez-Permouth, When cyclic singular modules over a simple ring are injective, J. Algebra, 263 (2003), 188-192.

[12] F. Kasch, Modules and Rings, Academic Press, New York, 1982.

[13] C. Lomp, On semilocal modules and rings, Comm. Algebra, 27 (4) (1999), 1921-1935.

[14] G. O. Michler and O.E. Villamayor, On rings whose simple modules are injective, J. Algebra, 25 (1973), 185-201.

[15] S. H. Mohamed and B. J. Muller, Continuous and Discrete Modules, Cambridge University Press, Cambridge, 1990.

[16] W. K. Nicholson and M.F. Yousif, Mininjective rings, J. Algebra, 187 (1997), 548-578.

[17] W. K. Nicholson and M.F. Yousif, Quasi-Frobenius Rings, Cambridge Tracts in Math., 158, Cambridge University Press, Cambridge, 2003.

[18] B. L. Osofsky, A generalization of quasi-Frobenius rings, J. Algebra, 4 (1966), 373387. 
[19] Liang Shen and Jianlong Chen, New characterizations of quasi-Frobenius rings, Comm. Algebra, 34 (2006), 2157-2165.

[20] P. F. Smith, CS-modules and weak CS-modules, Noncommutative Ring Theory, Springer LNM, 1448 (1990), 99-115.

[21] P. Vamos, The dual of the notion of 'finitely generated', J. London Math. Soc., 43 (1968), 186-209.

[22] M. F. Yousif, Y. Zhou and N. Zeyada, On pseudo-Frobenius rings, Canad. Math. Bull., 48 (2) (2005), 317-320.

[23] N. Zeyada, S. Hussein and A. Amin, Rad-injective and almost-injective modules and rings, Algebra Colloq., 18 (3) (2011), 411-418.

[24] N. Zeyada and N. Jarboui, s-CS modules and rings, Int. J. Algebra, 7 (2) 2013, 49-62.

(Received: July 1, 2015)

(Revised: January 6, 2016)
Nasr A. Zeyada

Cairo University

Faculty of Science

Department of Mathematics Egypt

and

University of Jeddah

Faculty of Science

Department of Mathematics

Saudi Arabia

nzeyada@sci.cu.edu.eg

Amr K. Amin

Beni-Suef University

Faculty of Science

Departement of Mathematics

Egypt

and

Umm Al-Qura University Jamoum University College Department of Mathematics Makkah, Saudi Arabia akgadelrab@uqu.edu.sa 